Educación Física y Ciencia, abril-junio 2018, vol. 20, n² 2, e046. ISSN 1514-0105 Universidad Nacional de La Plata

Facultad de Humanidades y Ciencias de la Educación

Centro de Historia Argentina y Americana

\title{
Efectos del entrenamiento de la aptitud muscular sobre la adiposidad corporal y el desempeño motriz en niños y jóvenes: un meta-análisis.
}

\author{
Adrián Casas \\ Departamento de Educación Física, Facultad de Humanidades y \\ Ciencias de la Educación. Universidad Nacional de La Plata. Argentina. \\ profcasas@yahoo.com.ar \\ Fernando Naclerio \\ Department of Life and Sport Science, University of Greenwich, United \\ Kingdom. \\ Xurxo Dopico Calvo \\ Departamento de Educación Física y Deportiva. Universidad \\ Da Coruña, España. \\ Christian García \\ Departamento de Investigación Francisco Valsecchi. Pontificia \\ Universidad Católica Argentina.
}

Recibido: 08 febrero 2018 - Aceptado: 15 marzo 2018 - Publicado: 27 abril 2018

(c) (i) (3) (2) Esta obra está bajo licencia Creative Commons Atribución-NoComercial-CompartirIgual 4.0 Internacional cc) 


\title{
Efectos del entrenamiento de la aptitud muscular sobre la adiposidad corporal y el desempeño motriz en niños y jóvenes: un meta-análisis.
}

\author{
Effects of Resistance Training on body fat and motor performance skills in children and adolescents: A Meta- \\ Analysis. \\ Adrián Casas \\ Departamento de Educación Física, Facultad de \\ Humanidades y Ciencias de la Educación. Universidad \\ Nacional de La Plata, Argentina \\ profcasas@yahoo.com.ar \\ Fernando Naclerio \\ Department of Life and Sport Science, University of \\ Greenwich, Reino Unido \\ Xurxo Dopico Calvo \\ Departamento de Educación Física y Deportiva. \\ Universidad Da Coruña, España \\ Christian García \\ Departamento de Investigación Francisco Valsecchi. \\ Pontificia Universidad Católica Argentina, Argentina
}

\section{RESUMEN:}

El propósito de este meta-análisis fue determinar la eficacia del efecto de entrenamiento de la aptitud muscular sobre el nivel de adiposidad y el rendimiento motriz en niños y jóvenes que no participan en deporte profesional y de alta competición. Se realizó una búsqueda sistemática en siete bases de datos electrónicas. Los estudios analizados fueron solo ensayos controlados que evaluaron los efectos del entrenamiento de la aptitud muscular sobre la adiposidad corporal (índice de masa corporal IMC; grasa corporal total GCT y adiposidad abdominal) y el desempeño motriz (salto en longitud sin carrera previa SL y lanzamiento de balón medicinal LMB) de niños y jóvenes. Dos evaluadores revisaron los estudios de forma independiente. Veintidós estudios cumplieron con los criterios de inclusión y fueron utilizados en este meta-análisis $(\mathrm{n}=697)$. El tamaño del efecto (TE) fue calculado usando el estadístico de Cohen, índice de la familia d. El efecto global estimado para el IMC fue pequeño y el límite del intervalo superior fue $>0(n=7, d=-0.12$, IC $95 \%-0.35$ a 0.10$)$. En el caso de la grasa corporal el TE fue moderado $(n=8, d=-0.37$, IC $95 \%-0.58$ a -0.17), al igual que el rendimiento de saltabilidad $(n=12, d=0.54$, IC 95\% 0.36 a 0.72 ) y lanzamiento $(n=4, d=0.49$, IC $95 \%$ 0.22 a 0.76). Los resultados de este meta-análisis sugieren que los programas de entrenamiento de la aptitud muscular son eficaces para reducir la adiposidad corporal e incrementar el desempeño motriz en la saltabilidad y el lanzamiento de niños y jóvenes que no participan en deporte profesional y de alta competición.

Palabras Clave: Aptitud muscular, Niños, Salud, Adiposidad, fuerza muscular.

\section{Abstract:}

The purpose of this meta-analysis was to evaluate the effectiveness of resistance training on the body fat percentage and motor skill performance in children and adolescents who do not participate in professional competitive sports. Systematic searches were undertaken in seven electronic databases. The studies under review consisted of controlled trials evaluating the effect of resistance training on body fat (Body Mass Index BMI, Total Body Fat TBF and central adiposity) and motor skill performance (standing long jump SLJ and medicine ball toss MBT) in children and adolescents. Two reviewers performed eligibility assessment independently, establishing that twenty-two studies met the inclusion criteria for meta-analysis $(\mathrm{n}=697)$. The effect size $(\mathrm{ES})$ was calculated using Cohen's d. Overall weighted ES for BMI was small with a confidence interval upper limit $>0(n=7, d=-0.12$, CI $95 \%-0.35$ to 0.10$)$. ES for body fat was moderate $(n=8, d=-0.37$, CI $95 \%-0.58$ to -0.17$)$. The same occurred with jumping performance $(n=12, d=0.54$, CI $95 \% 0.36$ to 0.72$)$ and throwing $(n=4, d=0.49$, CI $95 \% 0.22$ to 0.76$)$. Resistance training seems to be effective to reduce body fat and enhance motor skill performance in children and adolescents who do not participate in competitive sports. 
KEYWORDS: Muscular fitness, children, health, adiposity, muscular strength.

\section{INTRODUCCIÓN}

Las investigaciones relacionadas con los efectos positivos del ejercicio sobre la salud se desarrollaron tradicionalmente sobre la base del ejercicio aeróbico y el desarrollo de la aptitud cardio-respiratoria (Ortega et al., 2012; Ortega, Ruiz, Castillo, \& Sjostrom, 2008). La literatura científica constata que los programas de entrenamiento de la fuerza muscular con niños y jóvenes son seguros y eficaces cuando son supervisados por profesionales cualificados (Faigenbaum et al., 2009; Lloyd et al., 2014; Stratton et al., 2004). El desarrollo de este tipo de programas provoca adaptaciones positivas para la salud actuando sobre la modificación de la composición corporal (Schwingshandl, Sudi, Eibl, Wallner, \& Borkenstein, 1999), la reducción de la grasa corporal (Benson, Torode, \& Singh, 2008b; Watts et al., 2005) y el contenido mineral óseo (Faigenbaum \& Myer, 2010; Vicente-Rodríguez, 2006). Además, los programas de entrenamiento de la fuerza muscular producen mejoras significativas en el desarrollo de la fuerza, la potencia, la resistencia muscular local (Behringer, VomHeede, Yue, \& Mester, 2010; Faigenbaum et al., 2009; Falk \&Tenenbaum, 1996; Payne, Morrow, Johnson, \& Dalton, 1997; Sander, Keiner, Wirth, \& Schmidtbleicher, 2013) y el rendimiento motor general de niños y jóvenes. La evidencia actual sugiere que estos beneficios son independientes del desarrollo de la aptitud cardio-respiratoria (Castillo-Garzón, Ruiz, Ortega \& Gutierrez-Sainz, 2006; Ortega et al., 2008). Por lo tanto, se recomienda de manera específica la implementación de programas de entrenamiento de la aptitud muscular para niños y jóvenes.

Algunas revisiones sistemáticas han investigado los efectos de los programas de entrenamiento de la fuerza muscular sobre la salud en niños y jóvenes (Behringer et al., 2010; Benson, Torode, \& Singh, 2008a; Schranz, Tomkinson, \& Olds, 2013; Smith et al., 2014), sin embargo no conocemos estudios que hayan investigado la influencia de estos programas específica y conjuntamente sobre la adiposidad corporal y el rendimiento motriz en población infanto-juvenil. Por lo tanto, el propósito de esta revisión es determinar la eficacia del efecto de entrenamiento de la aptitud muscular sobre el nivel de adiposidad y el desempeño motriz en niños y jóvenes.

Terminología.

Definiremos a continuación la terminología que utilizaremos a lo largo de esta revisión sistemática. El término "aptitud muscular" representa en un solo concepto a la fuerza muscular, la resistencia muscular local y la potencia muscular (Smith et al., 2014). En este estudio, "entrenamiento de la aptitud muscular" será utilizado como sinónimo de entrenamiento de la fuerza muscular. El concepto de "desempeño motriz" expresa el nivel de desarrollo y rendimiento del movimiento humano, definido básicamente por el dominio de habilidades elementales (Stodden, 2008). Para los niños y jóvenes las actividades cotidianas, los juegos y deportes requieren, desde edades tempranas, habilidades motrices esenciales (como correr, saltar y lanzar), tanto para su funcionamiento biológico como social. El presente manuscrito se focalizará en los saltos y lanzamientos. El término "niños" será empleado para referirse a niñas y niños hasta 11 y 13 años respectivamente, mientras que el término "jóvenes" se referirá a mujeres y varones de 12 y 14 hasta 18 años, respectivamente (Faigenbaum et al., 2009). Finalmente, usaremos el término "adiposidad corporal" para referirnos a la cantidad de tejido adiposo valorado mediante diferentes métodos, técnicas e índices con validez científica reconocida (Absorciometría con rayos x de doble energía DEXA; Resonancia magnética nuclear; IMC; Medición de pliegues cutáneos; Circunferencia de cintura; Otros índices) (Ellis, 2000; KauferHorwitz \& Toussaint, 2008; Martín Moreno, Gómez Gandoy, \& Antoranz González, 2001); y usaremos el témino "peso saludable" para referirnos al peso corporal comprendido entre los percentiles $5 \mathrm{y}<85$ (Centers for Disease Control and Prevention, 2015; Hruby et al., 2012). 


\section{METODOLOGÍA}

Los métodos de análisis y los criterios de inclusión fueron especificados en un documento previo que se inscribió en el Registro Internacional Prospectivo de Revisiones Sistemáticas, PROSPERO (CRD42016032650).

La revisión sistemática de la literatura se realizó siguiendo las recomendaciones y criterios provistos por la Guía para informes de revisiones sistemáticas y meta-análisis: Declaración PRISMA (Liberati et al., 2009; Moher, Liberati, Tetzlaff, Altman, \&The PRISMA Group, 2009). Los procedimientos desarrollados en este meta-análisis se acordaron con antelación entre los autores e incluían: identificación; detección; elegibilidad; inclusión y exclusión de los estudios. Se realizó una búsqueda sistemática en siete bases de datos electrónicas (PubMed; Medline Plus; Scopus; SPORTDiscus; Cochrane Library; CINAHL y Web of Science); además, se realizaron búsquedas manuales en las listas de referencias bibliográficas de artículos científicos relevantes como revisiones sistemáticas y otros meta-análisis. La búsqueda de los artículos se realizó entre enero y febrero del año 2015 (no se incluyeron estudios posteriores a ese período). Se identificaron artículos escritos solo en idioma inglés y publicados en revistas científicas con sistema de revisión de pares; no se incluyó "literatura gris" (es decir, literatura que no ha sido publicada formalmente) (Hopewell, McDonald, Clarke, \&Egger, 2007).

Para la búsqueda se utilizaron las siguientes palabras claves y operadores booleanos: (strength OR endurance OR power OR "resistance training" OR "weight training" OR "muscular fitness") AND (children OR "elementary school" OR "primary school" OR young OR adolescents) AND ("body mass index" OR "body fat" OR "body weight" OR adiposity OR obesity OR overweight OR fatness OR "waist circumference") AND ("motor performance" OR "gross motor skills" OR "motor proficiency") AND (health OR risk OR benefit OR effect) AND ("controlled trial” OR random OR intervention OR prospective OR trial).

Una vez recogidas todas las referencias se combinaron los resultados de las búsquedas y se excluyeron aquellas que estaban duplicadas. Finalmente, se escogieron los estudios que cumplían con los criterios de elegibilidad.

Criterios de inclusión y exclusión.

Los criterios de inclusión para esta revisión sistemática fueron los siguientes:

1. Los participantes de los estudios debían ser niña/os y jóvenes de la población general, con edades entre 6 y 18 años, y cualquiera que fuese su Índice de Masa Corporal (IMC). Fueron excluidos los estudios en que participaron poblaciones de atletas de alto rendimiento y grupos con patologías cardiovasculares, respiratorias, metabólicas, neuromusculares y mentales.

2. Los estudios debían ser ensayos controlados, es decir, que comparasen uno o más grupos de intervención con uno o más grupos de control.

3. Los estudios debían utilizar una evaluación cuantitativa de la aptitud muscular (AM), el desempeño motriz general (DMG) y la adiposidad corporal (AC). Debían proporcionar claramente una descripción del programa de intervención y sus resultados o efectos logrados.

4. Fueron incluidos también estudios que comparaban el efecto de diversas intervenciones combinadas (por ejemplo, recomendaciones nutricionales; ejercicio aeróbico; ejercicio de fuerza; intervenciones psicológicas; etc.) siempre que la conformación de los subgrupos de intervención y control permitiesen identificar y comparar los efectos que este meta-análisis pretende estudiar.

5. Los estudios debían presentar información suficiente para el cálculo de la diferencia de medias entre los grupos.

6. Fueron incluidos solamente estudios publicados en inglés. 
Los criterios enunciados sustentan el concepto de que la diferencia encontrada entre grupo experimental y contraste es producida por la intervención.

Análisis estadístico.

El meta-análisis se realizó utilizando el software STATA 12.0 (StataCorp LP, USA). Se decidió utilizar el modelo de efectos aleatorios para computar los cálculos, debido a que en este se tiene en cuenta no solo la variabilidad intra-estudio sino también la variabilidad inter-estudios. Asimismo, las estimaciones fueron realizadas ponderando los resultados por la inversa de la varianza, y utilizando un intervalo de confianza al 95\%. Para la recolección de los datos se utilizaron los valores medio pre-test y post-test, las desviaciones estándar y el tamaño de la muestra, tanto del grupo contraste como del grupo experimental. El TE fue calculado usando el estadístico de Cohen (1988) denominado índice de la familia d, que es utilizado cuando se estima el tamaño del efecto en variables continuas; se calcula de la siguiente manera:

Donde es la media del grupo tratado, es la media del grupo contraste, y es la desviación conjunta de los dos grupos.

El entrenamiento de la aptitud muscular, según la acepción publicada en el documento de Consenso Internacional (Lloyd et al., 2014), fue considerado tratamiento experimental para el presente meta-análisis.

Los estudios integrados se evaluaron por medio del análisis de sensibilidad, en el cual se re-estima el TE tantas veces como estudios haya incluidos, eliminando en cada estimación los estudios uno por uno, de manera que se pueda evaluar la influencia de cada estudio en la variabilidad del TE. Los TE fueron evaluados usando la interpretación de Cohen para la cual un TE de 0.2 es pequeño, 0.5 mediano y 0.8 grande (Cohen, 1992).

Por último, se examinó la presencia de estudios con valores residuales atípicos $( \pm 1,96)$. El sesgo de publicación fue evaluado mediante los gráficos funnel plot, en los cuales se sitúa el TE en el eje de abscisas y la inversa del error estándar en el eje de coordenadas. Para descartar sesgo de publicación, el error estándar debería variar aleatoriamente alrededor del TE. También se realizó un análisis de las variables moderadoras que podría impactar en la variabilidad de los TE; considerando la cantidad de estudios disponibles se utilizó el análisis de meta-regresión bivariada entre la variable dependiente (TE en IMC; TE en Grasa corporal; TE en Saltabilidad y TE en Lanzamiento) y las variables moderadoras (edad; sexo; peso corporal; duración del programa; intensidad y frecuencia de entrenamiento), y la significación estadística se estableció en $\mathrm{p}<0.05$ para todos los casos.

\section{RESULTADOS}

La figura 1 muestra un diagrama de la estrategia de selección de los estudios. La búsqueda preliminar identificó 697 artículos científicos, se detectaron 89 estudios que estaban duplicados y fueron excluidos.

Se revisaron los 608 artículos restantes, de los cuales 573 estudios se excluyeron luego de la fase de lectura de los títulos y resúmenes. Fueron sugeridos para el análisis del texto completo 35 trabajos. Dos revisores expertos realizaron la lectura pormenorizada de los 35 artículos y se excluyeron 13. Finalmente 22 estudios (Benson et al., 2008b; Davis et al., 2009a; Davis et al., 2009b; Faigenbaum et al., 1996; Faigenbaum, Milliken, Moulton, \& Westcott, 2005; Faigenbaum, Zaichkowsky, Westcott, Micheli, \& Fehlandt, 1993; Faigenbaum \& Mediate, 2006; Faigenbaum et al., 2015; Faigenbaum et al., 2002, 2011; Falk \& Mor, 1996; Flanagan et al., 2002; Ingle, Sleap, \& Tolfrey, 2006; Kotzamanidis, 2006; Lee et al., 2012; McKay et al., 2000; Nichols, Sanborn \& Love, 2001; Schranz, Tomkinson, Parletta, Petkov, \& Olds, 2014; Shaibi et al., 2006; Treuth, Hunter, Figueroa-Colon, \& Goran, 1998; Viciana, Mayorga-Vega, \& Cocca, 2013; Yu et al., 2005) fueron incluidos en este meta-análisis. 


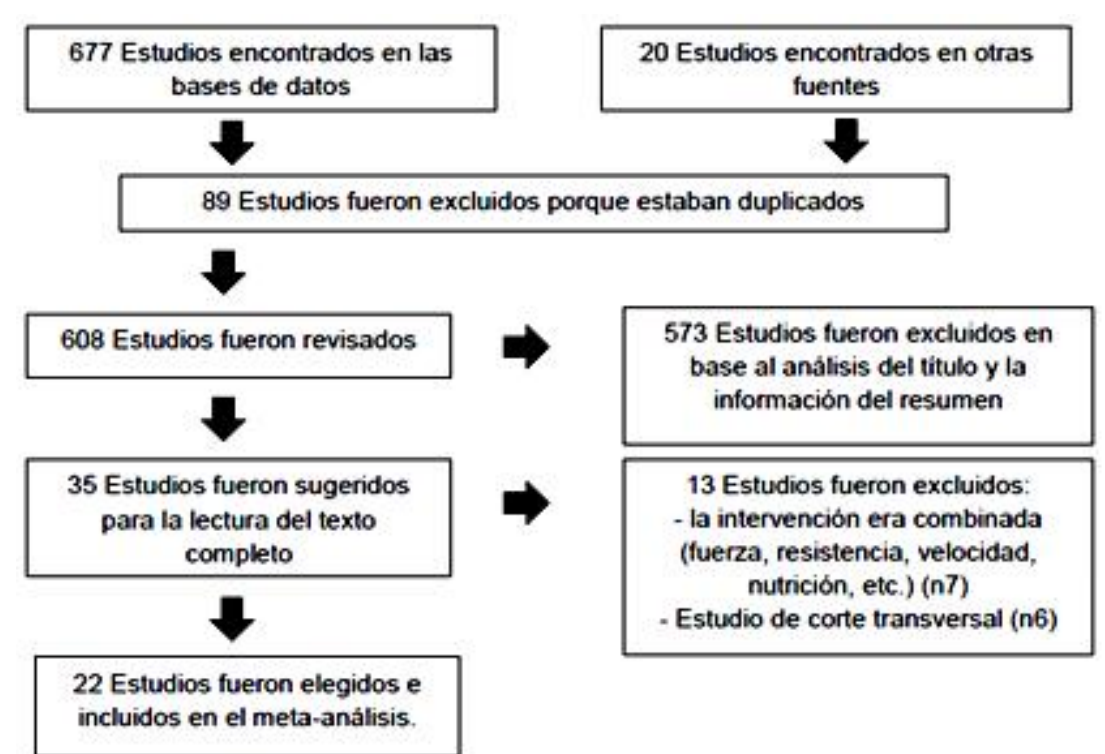

Figura 1.

Diagrama de flujo de búsqueda de los estudios.

Características de los estudios incluidos.

En el total de los 22 estudios incluidos en este meta-análisis hay 1135 participantes, 476 mujeres y 659 varones distribuidos en 44 grupos (intervención y contraste). Los estudios fueron publicados entre los años 1993 y 2015. La tabla 1 muestra las características más importantes de los estudios. 
Educación Física y Ciencia, abril-junio 2018, vol. 20, nº 2, e046. ISSN 1514-0105

\begin{tabular}{|c|c|c|c|c|}
\hline $\begin{array}{c}\text { Estudio } \\
\text { (autor/año) }\end{array}$ & Participantes & $\begin{array}{c}\text { Intervención } \\
\text { (tipo/duración) }\end{array}$ & $\begin{array}{l}\text { Variables } \\
\text { estudiad as }\end{array}$ & Efectos \\
\hline $\begin{array}{l}\text { Benson et al. } \\
\text { (2008b) }\end{array}$ & $\begin{array}{c}\mathrm{n}=79(33 ? / 46 ?) \\
12,3 \text { años }(=1,3) \\
\mathrm{n}=37 \mathrm{GI} / 4 \mathrm{GC}\end{array}$ & $\begin{array}{c}11 \text { ejercicios } x 8 \\
\text { rep. } X 2 \text { ser. } x 2 \\
\text { ses sem } x 8 \\
\text { semanas. PL y } \\
\text { manc. } \\
\text { Intensidad } 70 \text { - } \\
75 \% 1 \text { MR(PSE) }\end{array}$ & AAb. & $\begin{array}{l}\text { ? Adiposidad abdominal } \\
\text { en GI vs GC. } \\
\text { ?CiC fue mayor en } \\
\text { quienes más incremento } \\
\text { de la fuerza relativa } \\
\text { tuvieron. }\end{array}$ \\
\hline $\begin{array}{l}\text { "Davis et al. } \\
\left(2009_{a}\right)\end{array}$ & $\begin{array}{c}\mathrm{n}=16 ? ; 15,2 \text { años } \\
( \pm 1,1) \mathrm{SP} \\
\mathrm{n}=9 \mathrm{GI} / 7 \mathrm{GC}\end{array}$ & $\begin{array}{l}5 \text { e jercicios } \times 10-15 \\
\text { rep } x 2-3 \operatorname{ser} \times 2 \\
\text { ses sem x } 16 \\
\text { semanas. PL y MF. } \\
\text { Intensidad } 60 \text { - } \\
\quad 90 \% 1 \text { MR }\end{array}$ & $\begin{array}{l}\text { Adiposidad } \\
\text { (BMI) y PM. }\end{array}$ & $\begin{array}{c}\text { Sin diferencias } \\
\text { significativas en el BMI. }\end{array}$ \\
\hline $\begin{array}{l}{ }^{*} \text { Davis et a1. } \\
(2009 \mathrm{~b})\end{array}$ & $\begin{array}{c}\mathrm{n}=33(16 ? / 17 ?) \\
15,5 \text { años }(=1,0) \mathrm{SP} \\
\mathrm{n}=17 \mathrm{GI} / 16 \mathrm{GC}\end{array}$ & $\begin{array}{c}5 \text { ejercicios } \times 10-15 \\
\text { rep. } 2-3 \text { ser } \times 2 \\
\text { ses sem x } 16 \\
\text { semanas. PL y MF } \\
\text { Intensidad } 60 \text { - } \\
90 \% 1 \text { MR }\end{array}$ & $\begin{array}{l}\text { Adiposidad } \\
\text { (BMI) y PM. }\end{array}$ & $\begin{array}{c}\text { Sin diferencias } \\
\text { significativas en el BMI. }\end{array}$ \\
\hline $\begin{array}{l}\text { Faigenb aum } \\
\text { et al. (1993) }\end{array}$ & $\begin{array}{c}\mathrm{n}=23(8 ? / 15 ?) ; 10.8 \\
\text { años }( \pm 0.5) \\
\mathrm{n}=14 \mathrm{GI} 9 \mathrm{GC} .\end{array}$ & $\begin{array}{c}5 \text { ejercicios x } 10-15 \\
\text { rep } x 3 \operatorname{ser} x \\
2 \text { ses } / \operatorname{sem} x 8 \\
\text { semanas. PL y MF } \\
\text { Intensidad } 50-75 \text { - } \\
100 \% 10 \text { MR }\end{array}$ & $\begin{array}{l}\text { SV, LMB y } \\
\text { Sum PC }\end{array}$ & $\begin{array}{c}\text { ?SV } 13,8 \% \text { vs } 7,7 \% \\
\text { ?LM } 4,0 \text { vs } 3,9 \% \\
\text { ? Sum PC } 2,3 \% \text { vs ? } ? 1,7 \%\end{array}$ \\
\hline $\begin{array}{l}\text { Faigenb aum } \\
\text { et al. (1996) }\end{array}$ & $\begin{array}{c}\mathrm{n}=24(10 ? / 14 ?) ; \\
10.8( \pm 0.3) \\
\mathrm{n}=15 \mathrm{GI} / 9 \mathrm{GC}\end{array}$ & $\begin{array}{l}5 \text { e jercicios x } 10-20 \\
\text { rep } x 3 \operatorname{ser} x \\
2 \text { ses sem x } 8 \\
\text { semanas. PL y MF } \\
\text { Intensidad } 60 \text { - } \\
\quad 65 \% 1 \mathrm{MR}\end{array}$ & $\begin{array}{l}\text { SV, 6MR y } \\
\text { Flex. }\end{array}$ & $\begin{array}{c}\text { ?SV } 1,4 \% \text { vs } 0.5 \% \\
\text { ?6MR } 41 \% \text { (pp) y } 53 \% \\
\text { (er). }\end{array}$ \\
\hline $\begin{array}{l}\text { Faigenbaum } \\
\text { et al. (2002) }\end{array}$ & $\begin{array}{c}\mathrm{n}=55(21 ? / 34 ?) ; 9,5 \\
\text { años }( \pm 0.4) \\
\mathrm{n}=42 \mathrm{GI} / 13 \mathrm{GC}\end{array}$ & $\begin{array}{c}12 \text { ejercicio } \times 10- \\
15 \text { rep. } x 1 \text { ser } x 1 \text { a } \\
2 \text { ses } \operatorname{sem} \times 8 \\
\text { semanas. MF } \\
\text { Intensidad } 60 \text { - } \\
65 \% 1 \mathrm{MR}(\mathrm{PSE})\end{array}$ & $\begin{array}{l}\text { 1MR, SL, SV } \\
\text { y Flex. Efecto } \\
\text { de la } \\
\text { frecuencia de } \\
\text { entrenamiento }\end{array}$ & $\begin{array}{l}\text { 21MR entrenando } 2 \\
\text { versus } 1 \text { sesión por } \\
\text { semana. }\end{array}$ \\
\hline
\end{tabular}

Tabla 1.

Características de los estudios incluidos en el meta-análisis. 


\begin{tabular}{|c|c|c|c|}
\hline Faigenbaum & $\mathrm{n}=43(23 ? / 20 ?)$ & 9 ejercicios $x 6$ a 20 & 1MR, SL, SV, Ambos modelos de \\
\hline et al. (2005) & $\begin{array}{l}10.4 \text { años }( \pm 1,0) \\
\mathrm{n}=31 \mathrm{GI} / 2 \mathrm{GC}\end{array}$ & $\begin{array}{c}\text { rep.x } 1 \text { ser } x 2 \\
\text { ses sem } x 8 \\
\text { semanas. MF. } \\
\text { Intensidad } 50 \text { - } \\
\text { 80\%1MR. }\end{array}$ & $\begin{array}{l}\text { Flex. Efecto entrenamiento (Alto y } \\
\text { del número de Bajo numero de } \\
\text { repeticiones. repeticiones)? 1MR. }\end{array}$ \\
\hline Faigenbaum & $\mathrm{n}=118(41 ? / 77 ?)$ & 10 a 15 mimutos, 20 & ?SL $11 \%$ GI vs $1 \% \mathrm{GC}$ \\
\hline et al. (2006) & $\begin{array}{l}15,4 \text { años }(=0.5) \\
\mathrm{n}=69 \mathrm{GI} 49 \mathrm{GC}\end{array}$ & $\begin{array}{l}\text { a } 40 \text { ejercicios con } \\
\text { MB }(2 \mathrm{Kg}) \times 2 \\
\text { ses sem x } 6 \\
\text { semanas. } \\
\text { No informa } \\
\text { Intensidad }\end{array}$ & $\begin{array}{c}\text { ?LMB } 20 \% \mathrm{GI} \text { vs - } \\
1 \% \mathrm{GC} \text {. }\end{array}$ \\
\hline $\begin{array}{l}\text { Faigenb aum } \\
\text { et a1. (2011) }\end{array}$ & $\begin{array}{c}\mathrm{n}=40(24 ? / 16 ?) 7,5 \\
\text { años }( \pm 0.3) \\
\mathrm{n}=21 \mathrm{GI} 19 \mathrm{GC}\end{array}$ & $\begin{array}{c}15 \text { minutos, } 9 \\
\text { ejercicios de fuerza, } \\
\text { potencia y core } 7 \\
\text { a } 10 \text { rep x } 2 \text { ses/sem } \\
\text { x } 8 \text { semanas. No } \\
\text { informa Intensidad }\end{array}$ & $\begin{array}{cc}\text { FAb, SL, Rae, } & \text { ?SL } 8 \% \text { GI vs } \sin \\
\text { FBr } & \text { cambios GC } \\
& \text { ?FAb } 30 \% \text { GI vs } 1 \% \mathrm{GC} .\end{array}$ \\
\hline $\begin{array}{l}\text { Faigenbaum } \\
\text { et al. (2015) }\end{array}$ & $\begin{array}{c}\mathrm{n}=41(21 ? / 20 ?) 9,5 \\
\quad \text { años }(=0.3) \\
\mathrm{n}=20 \mathrm{GI} / 21 \mathrm{GC}\end{array}$ & $\begin{array}{c}15 \text { mimutos, } 6-7 \\
\text { estaciones de } \\
\text { fuerza, } \\
\text { lanzamientos, saltos } \\
\text { y core x } 2 \text { a } 3 \text { series } \\
\text { x } 2 \text { ses sem } x 8 \\
\text { semanas. } \\
\text { No informa } \\
\text { Intensidad }\end{array}$ & $\begin{array}{l}\text { Rae, SL, Flex, ?SL, FBr, FAbGI. } \\
\text { FBr, FAb }\end{array}$ \\
\hline $\begin{array}{c}\text { Falket al. } \\
(1996)\end{array}$ & $\begin{array}{c}\mathrm{n}=32 ? ; 6,7 \\
\text { años }(=0.4) \\
\mathrm{n}=17 \mathrm{GI} / 15 \mathrm{GC}\end{array}$ & $\begin{array}{l}40 \text { minutos de } \\
\text { ejercicios de fuerza } \\
\text { para brazos, piernas } \\
\text { y core, sin carga } \\
\text { externax } 2 \text { ses sem } \\
\text { x } 12 \text { semanas. } \\
\text { No informa } \\
\text { Intensidad. }\end{array}$ & $\begin{array}{r}\text { FAb, SL, Flex } \begin{array}{r}\text { ?FAb (26,4\%) SL } \\
(13,9 \%) \text { GI vs }-10.4 \mathrm{GC}\end{array}\end{array}$ \\
\hline
\end{tabular}

Tabla 1 (continúa). 
Educación Física y Ciencia, abril-junio 2018, vol. 20, n² 2, e046. ISSN 1514-0105

\begin{tabular}{|c|c|c|c|c|}
\hline $\begin{array}{l}\text { Flanagan et al. } \\
\text { (2002) }\end{array}$ & $\begin{array}{c}\mathrm{n}=58(30 ? / 28 ?) \\
8.5 \text { años }(=0.2) \\
\mathrm{n}=38 \mathrm{GI} / 20 \mathrm{GC}\end{array}$ & $\begin{array}{l}\text { Un grupo entrenó } \\
\text { con MF y otro con } \\
\text { el pe so corporal; } 9 \\
\text { ejercicios x } 10 \text { a } 15 \\
\text { rep, } x 2 \text { ses sem } x \\
10 \text { semanas. } \\
\text { Intensidad } 10 \text { a } 15 \\
\text { MR. }\end{array}$ & $\mathrm{LMB}, \mathrm{SL}$ & $\begin{array}{c}\text { Ambos grupos } \\
\text { intervención (MF y } \\
\text { Peso corporal) } \\
\text { ?significativamente } \\
\text { SL y LMB. }\end{array}$ \\
\hline Ingle et al. (2006) & $\begin{array}{c}\mathrm{n}=47 ? \quad 12,1 \\
\text { años }(=0.3) \\
\mathrm{n}=26 \mathrm{GI} / 21 \mathrm{GC}\end{array}$ & $\begin{array}{c}\text { 8 ejercicios } \\
\text { (Pliométricos y de } \\
\text { Fuerza) } \\
\text { combinando } \\
\text { intensidades (B aja } \\
\text { y Moderada) } x \\
3 \text { ses sem } x 12 \\
\text { semanas. } \\
70-100 \% \text { de } 10 \mathrm{MR}\end{array}$ & SL, SV y Sprint & $\begin{array}{l}\text { ?SL, SV y Sprint } \\
\text { en GI. }\end{array}$ \\
\hline $\begin{array}{l}\text { Kotzamanidis et } \\
\text { al. (2006) }\end{array}$ & $\begin{array}{c}\mathrm{n}=30 ? \\
11 \text { años }(=0.2) \\
\mathrm{n}=15 \mathrm{GI} / 15 \mathrm{GC}\end{array}$ & $\begin{array}{c}\text { Distintos tip os de } \\
\text { Saltos } \mathrm{x} 60 \text { a } 100, \\
\text { progresivos } 22 \\
\text { ses/sem } \times 10 \\
\text { semanas } \\
\text { Intensidad } \\
\text { Moderada-Alta }\end{array}$ & Sprint y SV & ?SV y Sprint GI. \\
\hline $\begin{array}{l}\text { "Lee et al. } \\
\text { (2012) }\end{array}$ & $\begin{array}{c}\mathrm{n}=29 ? \quad 14,5 \text { años } \\
(=1,5) \text { OB } \\
n=16 \mathrm{GI} / 13 \mathrm{GC}\end{array}$ & $\begin{array}{c}10 \text { ejercicios } \times 8 \text { a } \\
12 \text { rep } \times 2 \text { a } 3 \text { ser } x \\
3 \text { ses/sem } \times 12 \\
\text { semanas. MF } \\
\text { Intensidad } 60 \text { - } \\
75 \% 1 \mathrm{MR} \text {. }\end{array}$ & AAb y PM & $\begin{array}{c}\text { ?Adiposidad } \\
\text { abdominal GI vs } \\
\text { GC. }\end{array}$ \\
\hline $\begin{array}{l}\text { *McKay et a1. } \\
\text { (2000) }\end{array}$ & $\begin{array}{c}\mathrm{n}=144(74 ? / 70 ?) \\
8,9 \text { años }( \pm 0.3) \\
\mathrm{n}=63 \mathrm{GI} / 81 \mathrm{GC}\end{array}$ & $\begin{array}{c}10 \text { saltos diferentes } \\
\text { en series } \times 3 \\
\text { ses } \text { sem } \times 32 \\
\text { semanas. } \\
\text { No informa } \\
\text { Intensidad. }\end{array}$ & $\begin{array}{c}\text { DMO y \%Grasa } \\
\text { corporal }\end{array}$ & $\begin{array}{l}\text { Pequeña reducción } \\
\text { de la grasa corporal } \\
\text { en GI. }\end{array}$ \\
\hline
\end{tabular}

Tabla 1 (continúa). 


\begin{tabular}{|c|c|c|c|c|}
\hline $\begin{array}{l}\text { "Nichols et al. } \\
\text { (2001) }\end{array}$ & $\begin{array}{c}\mathrm{n}=67 ? \quad 15,7 \\
\text { años }( \pm 0.1) \\
\mathrm{n}=46 \mathrm{GI} / 21 \mathrm{GC}\end{array}$ & $\begin{array}{c}15 \text { ejercicios } x \text { a } \\
3 \text { series } x 9 \text { a } 15 \\
\text { rep } x 3 \text { ses sem } x \\
60 \text { semanas PL y } \\
\text { MF. } \\
\text { Intensidad } 10-15 \\
\text { MR }\end{array}$ & $\begin{array}{c}\text { Densidad mineral } \\
\text { osea, 1MR y } \\
\% \text { Grasa corporal }\end{array}$ & $\begin{array}{l}2 \% \text { grasa corporal } \\
\quad \text { GI } 20 \% \text {. }\end{array}$ \\
\hline $\begin{array}{c}\text { Schranz et al. } \\
\text { (2014) }\end{array}$ & $\begin{array}{c}\mathrm{n}=56 ? \text { SP y OB } \\
14,9 \text { años }( \pm 1,4) \\
\mathrm{n}=30 \mathrm{GI} / 26 \mathrm{GC}\end{array}$ & $\begin{array}{c}10 \text { ejercicios } \\
\text { poliarticulares } x 1 \\
\text { a } 3 \text { ser } \times 8 \text { a } 12 \text { rep } \\
\times 3 \text { ses sem } \times 24 \\
\text { semanas. PL y MF. } \\
\text { Intensidad } 70- \\
75 \% 1 \text { MR. }\end{array}$ & $\begin{array}{l}\text { Composición } \\
\text { corporal y Fuerza } \\
\text { (1MR) }\end{array}$ & $\begin{array}{l}\text { ?sum PC y \% grasa } \\
\text { ?masa libre de } \\
\text { grasa y 1MR GI }\end{array}$ \\
\hline $\begin{array}{c}\text { Shaibi et al. } \\
(2006)\end{array}$ & $\begin{array}{c}\mathrm{n}=22 ? 15,1 \\
\text { años }( \pm 0.5) \\
\mathrm{n}=11 \mathrm{GI} / 11 \mathrm{GC}\end{array}$ & $\begin{array}{c}5 \text { ejercicios x 3-15 } \\
\text { rep x 2-3 ser x } 2 \\
\text { ses sem x } 16 \\
\text { semanas. PL y MF } \\
\text { Intensidad } 60- \\
90 \% 1 \mathrm{MR}\end{array}$ & $\begin{array}{l}\text { \% grasa corporal, } \\
\text { Sensibilitad a la } \\
\text { Insulina y IMR }\end{array}$ & $\begin{array}{l}\text { ?sensibilidad a la } \\
\text { insulina y } 1 \text { MR GI } \\
\text { ?\% grasa corporal } \\
\text { ?masa libre de } \\
\text { grasa GI }\end{array}$ \\
\hline $\begin{array}{c}\text { Treuth et al. } \\
\text { (1998) }\end{array}$ & $\begin{array}{c}\mathrm{n}=22 ? \quad 8,5 \text { años } \\
( \pm 0.5) \mathrm{OB} \\
\mathrm{n}=11 \mathrm{GI} / 11 \mathrm{GC}\end{array}$ & $\begin{array}{c}7 \text { ejercicios de } \\
\text { fuerza x } 12 \text { a } 15 \\
\text { rep } \times 2 \text { a } 3 \text { ser } \times 3 \\
\text { ses } 3 \text { sem } \times 20 \\
\text { semanas. } \\
\text { Intensidad } 50 \text { - } \\
65 \% 1 \text { MR. }\end{array}$ & $\begin{array}{c}A A b, \text { Masa grasa, } \\
\text { MLG, TalG }\end{array}$ & $\begin{array}{l}\text { ?AAb y Masa } \\
\text { grasa de GI }\end{array}$ \\
\hline $\begin{array}{l}\text { Viciana et al. } \\
\text { (2013) }\end{array}$ & $\begin{array}{c}\mathrm{n}=75(33 ? / 42 ?) \\
11 \text { años }( \pm 0.8) \\
\mathrm{n}=38 \mathrm{GI} / 37 \mathrm{GC}\end{array}$ & $\begin{array}{c}8 \text { ejercicios } x 8 \text { a } \\
10 \text { rep } x 2 \operatorname{ser} \times 2 \\
\text { ses sem } x 8 \\
\text { semanas. } \\
\text { MB, Manc y BE. } \\
\text { Int. } 50-65 \% 1 \text { MR. }\end{array}$ & $\mathrm{SL}, \mathrm{FAb}$ & $\begin{array}{c}\text { ?fuerzay } \\
\text { resistencia } \\
\text { muscular local GI }\end{array}$ \\
\hline $\begin{array}{l}\text { Yu et al. } \\
(2005)\end{array}$ & $\begin{array}{c}\mathrm{n}=82(38 ? / 44 ?) \\
10.4 \text { años }(=1,0) \\
O B \\
\mathrm{n}=41 \mathrm{GI} / 41 \mathrm{GC}\end{array}$ & $\begin{array}{c}9 \text { ejercicios } x 1 \text { ser } \\
\times 10-20 \text { rep } \times 75 \% \\
(10 \text { MR }) \times 3 / \text { sem } 6 \\
\text { semanas } \\
\text { MF, PL y Manc. }\end{array}$ & $\begin{array}{c}\text { BMI, \% Grasa, } \\
\text { MLG }\end{array}$ & $\begin{array}{l}\text { ?MLG, ?\%Grasa } \\
\text { GI vs GC }\end{array}$ \\
\hline
\end{tabular}

Tabla 1 (continúa).

$\mathrm{GI}=$ grupo intervención; $\mathrm{GC}=$ grupo contraste; $\mathrm{Rep}=$ repeticiones; $\mathrm{Ser}=$ series; Ses/sem $=$ sesiones por semana; $\mathrm{PL}=$ pesos libres; $\mathrm{Manc}=$ mancuernas; $\mathrm{PSE}=$ percepción subjetiva de esfuerzo; $1 \mathrm{MR}=1$ máxima repetición; $\mathrm{AAb}=$ adiposidad abdominal; $\downarrow=$ reducción; $\mathrm{CiC}=$ circunferencia de cintura; $\mathrm{SP}=$ Sobrepeso; $\mathrm{BMI}=$ índice de masa corporal; $\mathrm{PM}=$ parámetros metabólicos; $\mathrm{MF}=$ máquinas de fuerza;

${ }^{*}$ Estudios con intervenciones $\mathrm{y} / \mathrm{u}$ objetivos combinados (nutrición/nutrición + fuerza/nutrición+ aeróbico/ etc.) que según los subgrupos de intervención permiten comparar efectos útiles para este metaanálisis.

$\mathrm{SV}=$ salto vertical; $\mathrm{LMB}=$ lanzamiento de medicine ball; Sum $\mathrm{PC}=$ sumatoria de pliegues cutáneos; $6 \mathrm{MR}=6$ máximas repeticiones; Flex= flexibilidad; $\mathrm{pp}=$ press de pecho, er= extensores de rodilla; $\mathrm{SL}=$ salto en longitud; $\mathrm{MB}=$ medicine ball; $\mathrm{FAb}=$ fuerza abdominal; $\mathrm{Rae}=$ resistencia aeróbica; $\mathrm{FBr}=$ fuerza de brazos; $\mathrm{OB}=$ obeso; $\mathrm{MLG}=$ masa libre de grasa; $\mathrm{DMO}=$ densidad mineral ósea; $\mathrm{TalG}=$ tolerancia a la glucosa; $\mathrm{BE}=$ bandas elásticas; $10 \mathrm{MR}=10$ máximas repeticiones.

Entrenamiento de la aptitud muscular, adiposidad corporal y desempeño motriz.

El efecto global estimado para el IMC fue pequeño y el límite del intervalo superior fue $>0(n=7, d=$ -0.12 , IC $95 \%-0.35$ a 0.10$)$. En el caso de la grasa corporal se observó un tamaño de efecto moderado( $\mathrm{n}=8$, $\mathrm{d}=-0.37$, IC 95\% -0.58 a -0.17), al igual que el rendimiento de saltabilidad ( $\mathrm{n}=12, \mathrm{~d}=0.54$, IC 95\% $0.36 \mathrm{a}$ 
0.72 ) y el lanzamiento ( $\mathrm{n}=4, \mathrm{~d}=0.49$, IC $95 \% 0.22$ a 0.76$)$. Se encontraron altos niveles de heterogeneidad en el análisis de los efectos del entrenamiento de la aptitud muscular sobre el $\operatorname{IMC}(\mathrm{Q}(6)=28,8$, $\mathrm{p}=0.00, \mathrm{I} 2=79,2 \%)$; la grasa corporal $(\mathrm{Q}(7)=104,31, \mathrm{p}=0.00, \mathrm{I} 2=93,3 \%)$ y el rendimiento de saltabilidad $(\mathrm{Q}(11)=114,31, \mathrm{p}=0.00, \mathrm{I} 2=90.1 \%)$; no ocurrió lo mismo con el caso de los efectos sobre el lanzamiento $(\mathrm{Q}(3)=3,13, \mathrm{p}=0.37, \mathrm{I} 2=4 \%)$.

Como muestran las figuras 2, 3 y 4, el entrenamiento de la aptitud muscular está asociado a la disminución de la grasa corporal y al incremento del rendimiento de la saltabilidad y del lanzamiento.

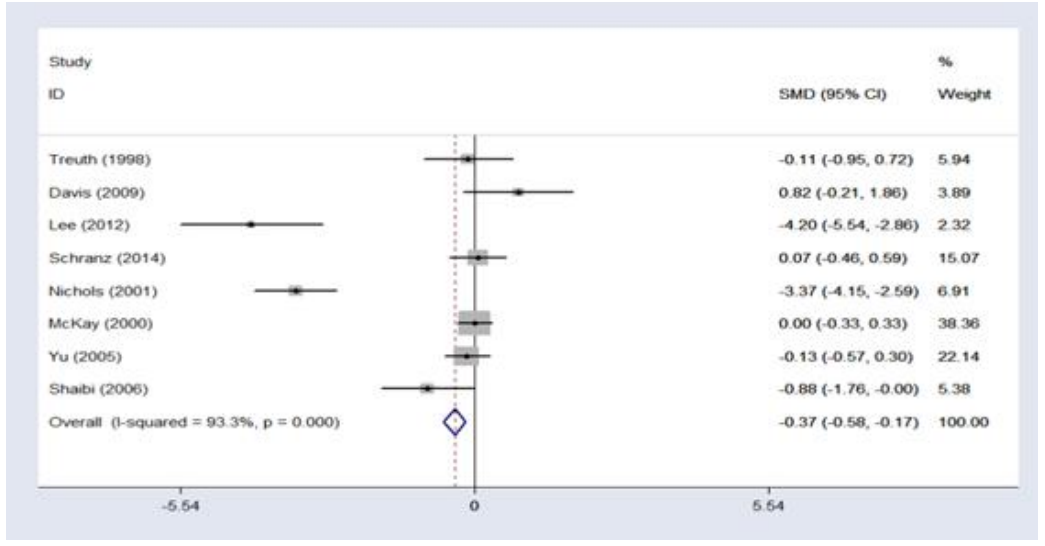

Figura 2.

Forest plot "Efectos del entrenamiento de la aptitud muscular sobre la grasa corporal en los estudios incluidos".

\begin{tabular}{|c|c|c|c|}
\hline \multicolumn{2}{|l|}{ Study } & \multicolumn{2}{|r|}{$\%$} \\
\hline ID & & SMD (95\% CI) & Weight \\
\hline Faigenbaum (2011) & $\rightarrow$ & $0.18(-0.44,0.81)$ & 8.38 \\
\hline Faigenbaum (2015) & $=$ & $1.38(0.70,2.07)$ & 6.90 \\
\hline Faigenbaum sv (1993) & $\div$ & $0.33(-0.51,1.18)$ & 4.56 \\
\hline Faigenbaum (2002) & $\rightarrow$ & $0.15(-0.47,0.78)$ & 8.36 \\
\hline Faigenbaum (2005) & $=$ & $0.24(-0.43,0.91)$ & 7.25 \\
\hline Faigenbaum (2006) & - & $0.33(-0.04,0.70)$ & 23.85 \\
\hline Faigenbaum sv (1996) & $\Leftrightarrow$ & $0.66(-0.19,1.51)$ & 4.50 \\
\hline Falk (1996) & $1 \div$ & $1.86(1.02,2.70)$ & 4.60 \\
\hline Flanagan (2002) & - & $0.55(0.00,1.10)$ & 10.67 \\
\hline Ingle (2006) & & $10.41(8.19,12.64)$ & 0.65 \\
\hline Kotzamanidis sv (2006) & $1 \div$ & $1.76(0.91,2.61)$ & 4.48 \\
\hline Viciana (2013) & - & $-0.08(-0.54,0.37)$ & 15.80 \\
\hline Overall (0-squared $=90,1 \%, p=0.000$ ) & 0 & $0.54(0.36,0.72)$ & 100.00 \\
\hline-12.6 & o & & \\
\hline
\end{tabular}

Figura 3.

Forest plot "Efectos del entrenamiento de la aptitud muscular sobre el rendimiento motriz de la saltabilidad en los estudios incluidos". 


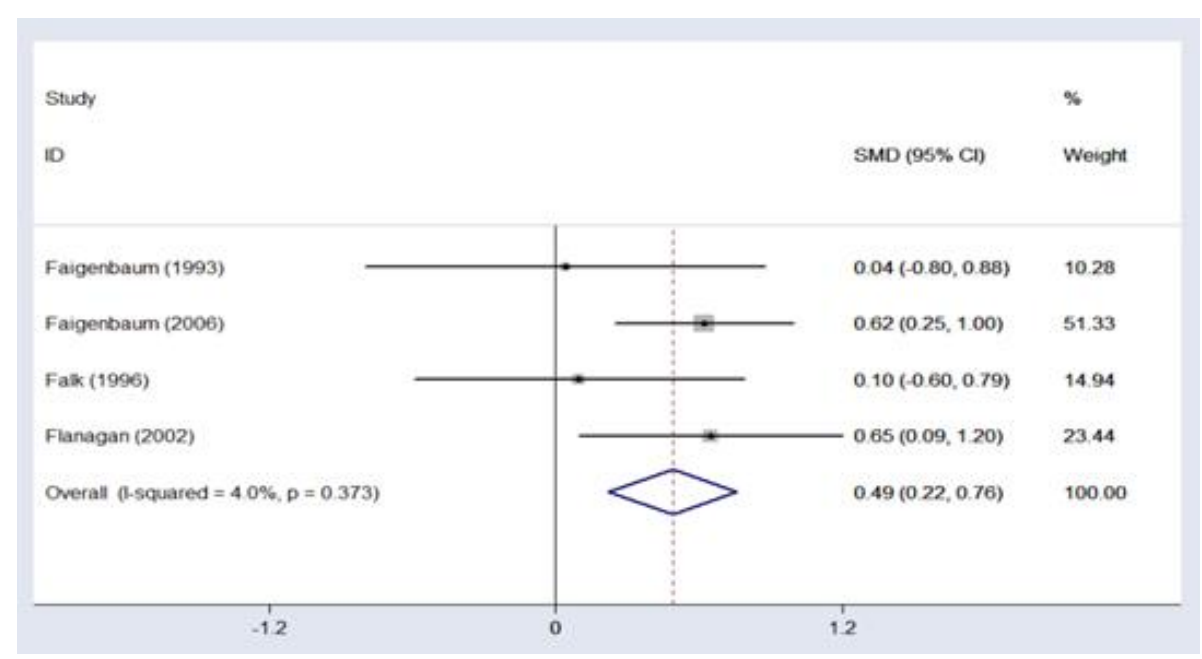

Figura 4.

Forest plot "Efectos del entrenamiento de la aptitud muscular sobre el rendimiento motriz del lanzamiento en los estudios incluidos".

Debido a la variabilidad de los resultados individuales se realizó un análisis de sensibilidad para re-estimar el tamaño del efecto global eliminando cada uno de los estudios y observando cómo influye en el efecto global cada uno de ellos. El análisis de sensibilidad de la grasa corporal indicó solo dos estudios (McKay et al., 2000; Nichols et al., 2001) con valores que exceden los intervalos de confianza $(-0.58$ y -0.17 , respectivamente) señalando que estos estudios tienen gran influencia en el tamaño del efecto global. Los demás análisis de sensibilidad indicaron que el tamaño del efecto global re-estimado se encuentra dentro de los intervalos de confianza, lo que sugiere que el análisis es robusto.

El análisis del funnel plot se mostró simétrico para el IMC, sin embargo, tanto para el TE en la grasa corporal y TE en el rendimiento de la saltabilidad se observó una pequeña asimetría hacia el lado izquierdo, mientras que para el TE en el rendimiento del lanzamiento la asimetría se manifiesta en sentido contrario. Finalmente, el análisis de las variables moderadoras (edad; sexo; peso corporal; duración del programa; intensidad y frecuencia de entrenamiento) sobre la variabilidad de los tamaños del efecto (TE en IMC; Grasa corporal; Saltabilidad y Lanzamiento) resultó estadísticamente significativo en tres casos: sexo (varón) y TE en la Saltabilidad ( $\mathrm{p}<0.035)$; duración del programa y TE en Saltabilidad $(\mathrm{p}<0.017)$ y frecuencia de entrenamiento y tamaño del efecto de Saltabilidad ( $\mathrm{p}<0.001)$.

\section{DISCUSIÓN}

El principal hallazgo de la presente revisión fue que los programas de entrenamiento de la aptitud muscular tienen un efecto positivo en la reducción de la adiposidad corporal y en el incremento del rendimiento motor expresado en la capacidad de salto y de lanzamiento en niños y jóvenes (figuras 2, 3 y 4). Los altos niveles de heterogeneidad encontrados en el análisis de los efectos del entrenamiento de la aptitud muscular sobre el IMC, la grasa corporal y el rendimiento de la saltabilidad, al igual que el bajo nivel de heterogeneidad en los efectos sobre el rendimiento del lanzamiento, podrían ser explicados por la pequeña cantidad de estudios meta-analizados, como lo sugieren algunos autores (Higgins, Thompson, Deeks, \& Altman, 2003), por lo cual, estos datos deberían ser tomados con precaución. Sin embargo, al revisar los resultados del análisis de la sensibilidad, se observa que el tamaño del efecto global re-estimado se encuentra dentro de los intervalos de confianza en la mayoría de los casos, lo que sugiere que el análisis es robusto. Los únicos dos estudios que exceden los intervalos de confianza (McKay et al., 2000; Nichols et al., 2001), se caracterizan por ser a la vez los estudios con mayor duración (32 y 60 semanas respectivamente) y mayor frecuencia de entrenamiento (tres sesiones por semana). 
La relación entre niveles elevados de grasa corporal y bajos rendimientos en la aptitud muscular ha sido respaldada por diversos estudios científicos (Benson et al., 2008a; Freitas et al., 2012; Hasselstrøm, Hansen, Froberg, \& Andersen, 2002; Nunez-Gaunaurd, Moore, Roach, Miller, \& Kirk-Sanchez, 2013; Ortega et al., 2008), sin embargo, los mecanismos implicados en esa relación son complejos y multivariados. De acuerdo con Wolfe (2006) el papel central que desempeña el músculo esquelético en el metabolismo de las proteínas de todo el cuerpo como depósito y lugar de síntesis proteica debe ser considerado con mayor detenimiento. Al mismo tiempo, el músculo esquelético es un tejido mecánico que requiere de la contracción muscular para su actividad; la falta de ésta altera el metabolismo muscular, provoca disfunción mitocondrial y es la génesis de diversas patologías metabólicas y crónicas (Wolfe, 2006; Barazzoni, 2015). El trabajo reciente de otros investigadores (Pedersen, 2013; Pedersen \& Febbraio, 2012) destaca al músculo esquelético como órgano de secreción de cientos de péptidos (secretoma muscular) con importante influencia e interacción sobre órganos y funciones vitales del cuerpo humano, entre ellos el tejido adiposo. La contracción muscular crónica provoca un incremento de la proteína PGC-1 alfa y de la Irisina, la cual actúa estimulando el tejido adiposo y modificando sus propiedades lipogénicas (Boström et al., 2012; Fisher et al., 2012; Pedersen \& Febbraio, 2012; Singhal et al., 2014), razones estas que podrían explicar algunos mecanismos actuantes en la interrelación que estamos analizando. Además, la reducción de los niveles de fuerza muscular, dinapenia (Clark \& Manini, 2012), fue señalada como un importante factor de riesgo para la salud. Este concepto se refiere a la pérdida de los niveles de fuerza muscular relacionada con causas no patológicas como por ejemplo enfermedades neuro-musculares. La dinapenia fue estudiada y desarrollada en adultos en los que se encontró que la pérdida de fuerza muscular se relaciona directamente con la reducción de la calidad de vida y el incremento de la mortalidad (Clark \& Manini, 2012). Los mecanismos para producir fuerza muscular combinan factores neurales con musculares, y la alteración y deterioro de cualquiera de ellos provoca dinapenia. Desde nuestra perspectiva, sugerimos revisar este concepto para poder aplicarlo en un espectro más amplio donde se consideren estudios con niños y jóvenes, ya que la evidencia actual indica que los niveles de fuerza muscular en niños en edad escolar se están reduciendo drásticamente (Cohen et al., 2011).

El meta-análisis de Smith (Smith et al., 2014) encontró un TE d=-0.25 para la relación entre aptitud muscular y adiposidad corporal de niños y jóvenes, siendo este resultado similar al de nuestro estudio. Existen algunas controversias relacionadas con el tipo de test empleado para valorar el rendimiento de la aptitud muscular; algunos autores recomiendan el uso de pruebas que no impliquen traslación, impulso o sostenimiento de la masa corporal, por ejemplo el test de fuerza de prensión de la mano (handgrip test), ya que con otras pruebas como el salto en longitud, salto vertical, las flexiones de brazos, etc., los resultados podrían ser influidos por la variación de la masa corporal y no tanto por los niveles de aptitud muscular. Mientras algunos autores señalan que los niños y jóvenes con sobrepeso presentan mayores niveles de masa magra respecto de sus pares sin sobrepeso (normopeso) (Deforche et al., 2003; Moliner-Urdiales et al., 2011) otros no encontraron esas diferencias (Artero et al., 2010; Riddiford-Harland, Steele, \& Baur, 2006). No obstante, la evidencia actual ha demostrado que los cambios en la aptitud muscular, medidos tanto en términos absolutos como relativos al peso corporal, se asocian inversamente con la adiposidad (Freitas et al., 2012; Hasselstrøm et al., 2002; Janz, Dawson \& Mahoney, 2002; Smith et al., 2014).

Otras revisiones sistemáticas y meta-análisis señalan que la mayor duración de la sesión y del programa de entrenamiento se asoció significativamente con cambios en la composición corporal (LeMura \& Maziekas, 2002; Maziekas, LeMura, Stoddard, Kaercher, \& Martucci, 2003). En nuestro estudio no encontramos significación estadística entre la duración del programa, el IMC y grasa corporal $(\mathrm{p}=0.77$ y $\mathrm{p}=0.40$, respectivamente); estos resultados diferentes pueden responder a que las revisiones sistemáticas citadas estudiaron los efectos del ejercicio aeróbico combinado con el entrenamiento de la fuerza muscular y sobre poblaciones de jóvenes obesos.

El meta-análisis de Schranz (2013) analizó los efectos del entrenamiento de la fuerza sobre la composición corporal y variables psico-sociales en niños y jóvenes con sobrepeso y obesidad. Los autores encontraron un 
TE $\mathrm{d}=-0.16$ y -0.24 ; ambos resultados son similares a los encontrados en nuestro estudio (TE-0.12 y - 0.37 para IMC y grasa corporal) al igual que la relación de estudios tratados.

La revisión sistemática de Benson (2008a) analizó los efectos del entrenamiento de la fuerza muscular sobre la adiposidad y otras variables de la aptitud metabólica (lipoproteínas, niveles de insulina y glucosa en sangre) de niños y jóvenes. Los investigadores destacan la importancia clínica de los resultados encontrados, al tiempo que señalan la poca cantidad de estudios relacionados con la temática y la diversidad de técnicas y metodologías empleadas para evaluar los efectos del entrenamiento sobre la grasa corporal. En esta línea, los resultados del presente estudio indicaron dificultades similares y aspectos relacionados con el diseño del programa de entrenamiento que pueden haber afectado a los resultados. Por ejemplo, el rango de la duración de los programas ( 6 a 60 semanas) y la edad de los participantes (8,5 a 15,7 años) deberían ser específicamente tomados en consideración. Los procesos de crecimiento, maduración y desarrollo de los participantes son muy dinámicos y heterogéneos, pudiendo influir significativamente en los resultados por sí mismos en rangos de tiempo tan amplios. Respecto a la edad de los participantes, el tejido adiposo celular subcutáneo responde a un patrón de comportamiento que demuestra un incremento entre los 6 y 10 años, siendo menor el incremento de la adiposidad en aquéllos niños que presentan mayores niveles de aptitud muscular (Lopes, Maia, Rodrigues, \& Malina, 2012).

El meta-análisis de Behringer et al.(2011) fue uno de los primeros en evaluar los efectos del entrenamiento de la aptitud muscular sobre el rendimiento motriz, siendo sus resultados $\mathrm{TE} d=0.54 ; 0.53$ y 0.99 para el rendimiento del salto, la carrera y lanzamiento, respectivamente. Nuestros resultados son similares a los encontrados en el rendimiento del salto y lanzamiento (TE 0.54 y 0.49 , respectivamente). Los investigadores encontraron mayor tamaño del efecto en subgrupos de no atletas respecto a los deportistas, lo que pondría de manifiesto la importancia del desarrollo de los mecanismos de control neuromusculares ligados al entrenamiento de la fuerza, los cuáles son más relevantes en aquellos niños o jóvenes sin experiencia en el entrenamiento de la fuerza muscular (Faigenbaum et al., 2009; Granacher et al., 2011; Payne et al., 1997; Yu et al., 2005). Como indica la tabla 1, los participantes de los estudios incluidos en esta revisión fueron niños y jóvenes sin participación en deportes de alta competición, por lo tanto bien podríamos explicar nuestros resultados a partir de las mejoras en los mecanismos neuromusculares (por ejemplo, incremento del reclutamiento de unidades motoras; aumento de la velocidad de conducción de los impulsos nerviosos y mejora de la coordinación intra e intermuscular) como respuesta al entrenamiento (Faigenbaum et al., 2009; Granacher et al., 2011). La evidencia indica que las adaptaciones neurales más significativas se producen en la fase inicial de los programas de entrenamiento (Bouchant, Martin, Maffiuletti, \& Ratel, 2011; Granacher et al., 2011; Kraemer, Fry, Frykman, Conroy, \& Hoffman, 1989; O’Brien, Reeves, Baltzopoulos, Jones, \& Maganaris, 2010; Ramsay et al., 1990).

Fortalezas y limitaciones.

La presente revisión sistemática tuvo las siguientes fortalezas: primero, la utilización de un estricto conjunto de criterios de inclusión/exclusión, ya que nuestro estudio sintetizó el resultado de 22 estudios que analizaron los efectos del entrenamiento de la fuerza muscular (empleando diversos tipos de estrategias) sobre la adiposidad corporal y el rendimiento motriz general de 1135 participantes de población infanto-juvenil de 7 países; segundo, que fueron evaluados solamente Ensayos Controlados (aleatorizados y no aleatorizados).

Las limitaciones que podemos señalar fueron: I) la pequeña cantidad de estudios disponibles, por ejemplo al evaluar los efectos del entrenamiento sobre el rendimiento motriz del lanzamiento (4 estudios) y II) el potencial sesgo de publicación de los estudios sugerido en el análisis del funnel plot.

Es necesaria una mayor cantidad de estudios que impliquen a niños pre-púberes, púberes y post-púberes (con diferentes estatus de peso corporal) para profundizar en el conocimiento de los mecanismos del ejercicio que optimizan estos efectos saludables. 


\section{CONCLUSIONES}

Los resultados de este meta-análisis sugieren que los programas de entrenamiento de la aptitud muscular son eficaces para reducir la adiposidad corporal e incrementar el desempeño motriz en la saltabilidad y el lanzamiento de niños y jóvenes que no participan en deporte profesional y de alta competición.

\section{ReFERENCIAS}

Artero, E. G., Espana-Romero, V., Ortega, F. B., Jimenez-Pavon, D., Ruiz, J. R., Vicente-Rodriguez, G., ... Castillo, M. J. (2010). Health-related fitness in adolescents: underweight, and not only overweight, as an influencing factor. The AVENA study. Scandinavian Journal of Medicine \& Science in Sports, 20(3), 418-427. http://doi.org/10.1 111/j.1600-0838.2009.00959.x.

Barazzoni, R. (2015) Muscle Biopsy To Investigate Mitochondrial Turnover. In: Luzi, L. Ed. Cellular Physiology and Metabolism of Physical Exercise. Springer Milan. 67-84. http://link.springer.com/chapter/10.1007/978-88-47 0-2418-2 8 .

Behringer, M., Vom Heede, A., Matthews, M., y Mester, J. (2011). Effects of strength training on motor performance skills in children and adolescents: a meta-analysis. Pediatric Exercise Science, 23(2), 186-206.

Behringer, M., Vom Heede, A., Yue, Z, y Mester, J. (2010) Effects of resistance training in children and adolescents: a meta-analysis. Pediatrics; 126:e1199-210.

Benson, A. C., Torode, M.E., y Singh, M. A. F. (2008a) The Effect of High-Intensity Progressive Resistance Training on Adiposity in Children: A Randomized Controlled Trial. Int J Obes; 32:1016-27. doi:10.1038/ijo.2008.5.

Benson, A. C., Torode, M. E., Singh, M. A. F.(2008b) Effects of resistance training on metabolic fitness in children and adolescents: a systematic review. Obes Rev; 9:43-66. doi:10.1111/j. 1467-789X.2007.00388.x.

Boström, P., Wu, J., Jedrychowski, M. P., Korde, A., Ye, L., Lo, J. C., ... Spiegelman, B. M. (2012). A PGC1- $\alpha-$ dependent myokine that drives brown-fat-like development of white fat and thermogenesis. Nature, 481(7382), 463-468. http://doi.org/10.1038/nature10777.

Bouchant, A., Martin, V., Maffiuletti, N. A., y Ratel, S. (2011). Can muscle size fully account for strength differences between children and adults? Journal of Applied Physiology, 110(6), 1748-1749. http://doi.org/10.1152/jappl physiol.01333.2010.

Castillo-Garzón, M. J., Ruiz, J. R., Ortega, F. B., y Gutierrez-Sainz, A. (2006). A Mediterranean Diet Is Not Enough for Health: Physical Fitness Is an Important Additional Contributor to Health for the Adults of Tomorrow. En A. P. Simopoulos \& F. Visioli (Eds.), World Review of Nutrition and Dietetics (pp. 114-138). Basel: KARGER. Recuperado a partir de http://www.karger.com/doi/10.1159/000097913.

Clark, B.C., y Manini, T.M. (2012) What is dynapenia? Nutr Burbank Los Angel Cty Calif; 28: 495-503. doi:10.1016/ j.nut.2011.12.002.

Cohen, D., Voss, C., Taylor, M., Delextrat, A., Ogunleye, A., y Sandercock, G. (2011). Ten-year secular changes in muscular fitness in English children. Acta paediatrica (Oslo, Norway\#: 1992), 100(10), e175-7. http:// doi.org/10.1111/j.1651-2227.2011.02318.x.

Cohen J. (1988) Statistical Power Analysis for the Behavioral Sciences. 2nd ed. New York, NY, US: Academic press.

Cohen, J. A power primer. Psychol Bull 1992; 112:155-9.

Davis, J., Kelly, L., Lane, C., Ventura, E., Byrd-Williams, C., Alexandar, K., ... Goran, M. (2009a). Randomized Control Trial to Improve Adiposity and Insulin Resistance in Overweight Latino Adolescents. Obesity (Silver Spring)., $17(8), 1542-1548$.

Davis, J., Tung, A., Chak, S., Ventura, E., Byrd-Williams, C., Alexander, K., ... Goran, M. (2009b). Aerobic and Strength Training Reduces Adiposity in Overweight Latina Adolescents. Med Sci Sports Exerc, 41((7)), 14941503. 
Deforche, B., Lefevre, J., De Bourdeaudhuij, I., Hills, A. P., Duquet, W., y Bouckaert, J. (2003). Physical fitness and physical activity in obese and nonobese Flemish youth. Obesity Research, 11(3), 434-441.

Ellis, K.J. (2000) Human Body Composition: In Vivo Methods. Physiol Rev; 80:649-80.

Faigenbaum, A., Westcott, W., Micheli, L., Outerbridge, A. R., Long, C., LaRosa-Loud, R., y Zaichkowsky, L. (1996). The effects of strength training and detraining on children. Journal Of Strength And Conditioning Research / National Strength \& Conditioning Association, 10(2), 109-114.

Faigenbaum, A. D., Milliken, L. A., Loud, R. L., Burak, B. T., Doherty, C. L., y Westcott, W. L. (2002). Comparison of 1 and 2 days per week of strength training in children. Research Quarterly for Exercise and Sport, 73(4), 416-424. http://doi.org/10.1080/02701367.2002.10609041

Faigenbaum, A., Milliken, L., Moulton, L., y Westcott, W. (2005). Early Muscular Fitness Adaptations in Children in Response to Two Different Resistance Training Regimens. Pediatric Exercise Science, (17), 237-248.

Faigenbaum, A., Zaichkowsky, L., Westcott, W., Micheli, L., y Fehlandt, A. (1993). The Effects of a Twice-a-Week Strength Training Program on Children. Pediatric Exercise Sciertce, 5, 339-346.

Faigenbaum, A. D., Bush, J. A., Mcloone, R. P., Kreckel, M. C., Farrell, A., Ratamess, N. A., y Kang, J. (2015). Benefits of strength and skill-based training during primary school physical education. Journal of Strength and Conditioning Research, 29(5), 1255-1262. http://doi.org/10.1519/JSC.0000000000000812.

Faigenbaum, A., y Mediate, P. (2006) Effects of Medicine Ball Training on Fitness Performance of High-School Physical Education Students. Phys Educ; 63:160-7.

Faigenbaum, A. D., Farrell, A., Fabiano, M., Radler, T., Naclerio, F., Ratamess, N. A., ... Myer, G. D. (2011). Effects of Integrative Neuromuscular Training on Fitness Performance in Children. Pediatric Exercise Science, 23(4), 573-584.

Faigenbaum, A. D., Kraemer, W.J., Blimkie, C. J., Jeffreys, I., Micheli, L. J., Nitka, M., y Rowland, T. W. (2009). Youth resistance training: updated position statement paper from the national strength and conditioning association. The Journal of Strength \& Conditioning Research, 23, S60-S79.

Faigenbaum, A. D. y Myer, G. D. (2010) Resistance training among young athletes: safety, efficacy and injury prevention effects. BrJ Sports Med; 44:56-63.

Falk, B., y Mor, G. (1996) The Effects of Resistance and Martial Arts Training in 6- to 8-Year Old Boys. Pediatr Exerc Sci; 8:48-56.

Falk B., y Tenenbaum, G. (1996) The effectiveness of resistance training in children. A meta-analysis. Sports Med Auckl $N Z ; 22: 176-86$.

Fisher, F. M., Kleiner, S., Douris, N., Fox, E. C., Mepani, R. J., Verdeguer, F., ... Spiegelman, B. M. (2012). FGF21 regulates PGC- $1 \alpha$ and browning of white adipose tissues in adaptive thermogenesis. Genes \& Development, 26(3), 271-281. http://doi.org/10.1101/gad.177857.111.

Flanagan, S., Laubach, L., De Marco Jr., G., Alvarze, C., Borchers, S., Dressman, E., ... Weseli, D. (2002). Effects of Two Different Strength Training Modes on Motor Performance in Children. Research Quarterly for Exercise and Sport, 73(3), 340-344.

Freitas, D., Beunen, G., Maia, J., Claessens, A., Thomis, M., Marques, A., ... Lefevre, J. (2012). Tracking of fatness during childhood, adolescence and young adulthood: a 7-year follow-up study in Madeira Island, Portugal. Annals of Human Biology, 39(1), 59-67.

Granacher, U., Goesele, A., Roggo, K., Wischer, T., Fischer, S., Zuerny, C., ... Kriemler, S. (2011). Effects and mechanisms of strength training in children. International journal of sports medicine, $32(5), 357$.

Hasselstrøm, H., Hansen, S. E., Froberg, K., y Andersen, L. B. (2002). Physical fitness and physical activity during adolescence as predictors of cardiovascular disease risk in young adulthood. Danish Youth and Sports Study. An eight-year follow-up study. International Journal of Sports Medicine, 23 Suppl 1, S27-31. http://doi.org/10.10 $55 / \mathrm{s}-2002-28458$

Higgins, J. P. T., Thompson, S. G., Deeks, J. J., y Altman, D. G. (2003). Measuring inconsistency in meta-analyses. BMJ: British Medical Journal, 327(7414), 557-560. 
Hopewell, S., McDonald, S., Clarke, M. J., y Egger, M. (2007). Grey literature in meta-analyses of randomized trials of health care interventions. En The Cochrane Collaboration (Ed.), Cochrane Database of Systematic Reviews. Chichester, UK: John Wiley \& Sons, Ltd. Recuperado a partir de http://doi.wiley.com/10.1002/14651858.M R000010.pub3.

Hruby, A., Chomitz, V. R., Arsenault, L. N., Must, A., Economos, C. D., McGowan, R. J., y Sacheck, J. M. (2012). Predicting Maintenance or Achievement of Healthy Weight in Children: The Impact of Changes in Physical Fitness. Obesity, 20(8), 1710-1717. http://doi.org/10.1038/oby.2012.13.

Ingle, L., Sleap, M., y Tolfrey, K. (2006) The effect of a complex training and detraining programme on selected strength and power variables in early pubertal boys.J Sports Sci; 24: 987-97. doi:10.1080/02640410500457117.

Janz, KF., Dawson, JD., y Mahoney, LT. (2002) Increases in physical fitness during childhood improve cardiovascular health during adolescence: The Muscatine Study. Int J Sports Med;23: S15-21.

Kaufer-Horwitz, M., y Toussaint, G. (2008) Indicadores antropométricos para evaluar sobrepeso y obesidad en pediatría. Bol Méd Hosp Infant México; 65:502-18.

Kotzamanidis, C. (2006) Effect of plyometric training on running performance and vertical jumping in prepubertal boys. J Strength Cond Res Natl Strength Cond Assoc; 20:441-5.

Kraemer, W. J., Fry, A. C., Frykman, P. N., Conroy, B., y Hoffman, J. (1989). Resistance training and youth. Recuperado a partir de http://kuscholarworks.ku.edu/handle/1808/11384.

Lee, S., Bacha, F., Hannon, T., Kuk, J. L., Boesch, C., y Arslanian, S. (2012). Effects of Aerobic Versus Resistance Exercise Without Caloric Restriction on Abdominal Fat, Intrahepatic Lipid, and Insulin Sensitivity in Obese Adolescent Boys A Randomized, Controlled Trial. Diabetes, 61(11), 2787-2795. http://doi.org/10.2337/db1 2-0214

LeMura, L.M., y Maziekas, M.T. (2002) Factors that alter body fat, body mass, and fat-free mass in pediatric obesity. Med Sci Sports Exerc; 34:487-96.

Liberati, A., Altman, D. G., Tetzlaff, J., Mulrow, C., Gøtzsche, P. C., Ioannidis, J. P. A., ... Moher, D. (2009). The PRISMA Statement for Reporting Systematic Reviews and Meta-Analyses of Studies That Evaluate Health Care Interventions: Explanation and Elaboration. PLoS Med, 6(7), e1000100. http://doi.org/10.1371/journal.pme d.1000100.

Lloyd, R. S., Faigenbaum, A. D., Stone, M. H., Oliver, J. L., Jeffreys, I., Moody, J. A., ... Myer, G. D. (2014). Position statement on youth resistance training: the 2014 International Consensus. British Journal of Sports Medicine, 48(7), 1-12.

Lopes, Ví. P., Maia, J. A. R., Rodrigues, L. P., y Malina, R. (2012). Motor coordination, physical activity and fitness as predictors of longitudinal change in adiposity during childhood. European Journal of Sport Science, 12(4), 384-391. http://doi.org/10.1080/17461391.2011.566368

Maziekas, M. T., LeMura, L. M., Stoddard, N. M., Kaercher, S., y Martucci, T. (2003). Follow up exercise studies in paediatric obesity: implications for long term effectiveness. British Journal of Sports Medicine, 37(5), 425-429.

McKay, H. A., Petit, M. A., Schutz, R. W., Prior, J. C., Barr, S. I., y Khan, K. M. (2000). Augmented trochanteric bone mineral density after modified physical education classes: a randomized school-based exercise intervention study in prepubescent and early pubescent children. The Journal of Pediatrics, 136(2), 156-162.

Moher, D., Liberati, A., Tetzlaff, J., Altman, D. G., y The PRISMA Group. (2009). Preferred Reporting Items for Systematic Reviews and Meta-Analyses: The PRISMA Statement. PLoS Med, 6(7), e1000097. http://doi.org/ 10.1371/journal.pmed.1000097.

Moliner-Urdiales, D., Ruiz, J. R., Vicente-Rodriguez, G., Ortega, F. B., Rey-Lopez, J. P., Espana-Romero, V., ... Moreno, L. A. (2011). Associations of muscular and cardiorespiratory fitness with total and central body fat in adolescents: The HELENA Study. British Journal of Sports Medicine, 45(2), 101-108. http://doi.org/10.1136/ bjsm.2009.062430.

Moreno, M., Gómez Gandoy, JB., y Antoranz González, MJ. (2001). Medición de la grasa corporal mediante impedancia bioeléctrica, pliegues cutáneos y ecuaciones a partir de medidas antropométricas. Análisis comparativo. Rev Esp Salud Pública; 75:221-36. 
Nichols, D. L., Sanborn, C. F., y Love, A. M. (2001) Resistance training and bone mineral density in adolescent females. J Pediatr; 139:494-500.

Nunez-Gaunaurd, A., Moore, J. G., Roach, K. E., Miller, T. L., y Kirk-Sanchez, N. J. (2013). Motor Proficiency, Strength, Endurance, and Physical Activity Among Middle School Children Who Are Healthy, Overweight, and Obese. Pediatric Physical Therapy, 25(2), 130-138. http://doi.org/10.1097/PEP.0b013e318287caa3.

O’Brien, T. D., Reeves, N. D., Baltzopoulos, V., Jones, D. A., y Maganaris, C. N. (2010). In vivo measurements of muscle specific tension in adults and children. Experimental Physiology, 95(1), 202-210. http://doi.org/10.1113/ expphysiol.2009.048967

Ortega, F. B., Ruiz, J. R., Castillo, M.J., y Sjostrom, M. (2008). Physical fitness in childhood and adolescence: a powerful marker of health. International Journal of Obesity, 32(1), 1-11. http://doi.org/10.1038/sj.ijo.0803774.

Ortega, F. B., Silventoinen, K., Tynelius, P., y Rasmussen, F. (2012). Muscular strength in male adolescents and premature death: cohort study of one million participants. BMJ, 345, 1-12. http://doi.org/10.1136/bmj.e7279.

Payne, V. G., Morrow, J. R., Johnson, L., y Dalton, S. N. (1997). Resistance training in children and youth: a meta-analysis. Research Quarterly for Exercise and Sport, 68(1), 80-88. http:// doi.org/10.1080/02701367.1997.10608869.

Pedersen, B. K. (2013) Muscle as a Secretory Organ. Compr Physiol; 3:1337-62. doi:10.1002/ cphy.c120033.

Pedersen, B. K., y Febbraio, M. A. (2012) Muscles, exercise and obesity: skeletal muscle as a secretory organ. Nat Rev Endocrinol; 8:457-65. doi:10.1038/nrendo.2012.49.

Physical Activity Guidelines - health.gov. (2008). http://health.gov/paguidelines/guidelines/ (accessed 15 Dec2015).

Ramsay, J. A., Blimkie, C. J., Smith, K., Garner, S., MacDougall, J. D., y Sale, D. G. (1990). Strength training effects in prepubescent boys. Medicine and Science in Sports and Exercise, 22(5), 605-614.

Riddiford-Harland, D. L., Steele, J. R., y Baur, L. A. (2006) Upper and lower limb functionality: Are these compromised in obese children? Int J Pediatr Obes 2006; 1:42-9.doi:10.1080/17477160 600586606.

Ruiz, J. R., Ortega, F. B., Wärnberg, J., Moreno, L. A., Carrero, J. J., Gonzalez-Gross, M., ... Sjöström, M. (2008). Inflammatory proteins and muscle strength in adolescents: The Avena study. Archives of Pediatrics \& Adolescent Medicine, 162(5), 462-468. http://doi.org/10.1001/archpedi.162.5.462

Sander, A., Keiner, M., Wirth, K., y Schmidtbleicher, D. (2013). Influence of a 2-year strength training programme on power performance in elite youth soccer players. European Journal of Sport Science, 13(5), 445-451. http:// doi.org/10.1080/17461391.2012.742572.

Schranz, N., Tomkinson, G., y Olds, T. (2013) What is the Effect of Resistance Training on the Strength, Body Composition and Psychosocial Status of Overweight and Obese Children and Adolescents? A Systematic Review and Meta-Analysis. Sports Med; 43:893-907.

Schranz, N., Tomkinson, G., Parletta, N., Petkov, J., y Olds, T. (2014). Can resistance training change the strength, body composition and self-concept of overweight and obese adolescent males? A randomised controlled trial. BrJ Sports Med, 48, 1482-1488.

Schwingshandl, J., Sudi, K., Eibl, B., Wallner, S., y Borkenstein, M. (1999). Effect of an individualised training programme during weight reduction on body composition: a randomised trial. Archives of Disease in Childhood, 81(5), 426-428. http://doi.org/10.1136/adc.81.5.426.

Shaibi, G. Q., Cruz, M. L., Ball, G. D. C., Weigensberg, M. J., Salem, G. J., Crespo, N. C., y Goran, M. I. (2006). Effects of resistance training on insulin sensitivity in overweight Latino adolescent males. Medicine and Science in Sports and Exercise, 38(7), 1208-1215. http://doi.org/10.1249/01.mss.000227304.88406.

Singhal, V., Lawson, E. A., Ackerman, K. E., Fazeli, P. K., Clarke, H., Lee, H., ... Misra, M. (2014). Irisin Levels Are Lower in Young Amenorrheic Athletes Compared with Eumenorrheic Athletes and Non-Athletes and Are Associated with Bone Density and Strength Estimates. PLoS ONE, 9(6). http://doi.org/10.1371/journal.pon e.0100218.

Smith, J., Eather, N., Morgan, P., Plotnikoff, R., Faigenbaum, A., y Lubans, D. (2014). The Health Benefits of Muscular Fitness for Children and Adolescents: A Systematic Review and Meta-Analysis. Sports Medicine, 44(9), 1209-1223. 
Start active, stay active: report on physical activity in the UK - Publications - GOV.UK. (2011). https://www.gov.uk/government/publications/start-active-stay-active-a-report-on-physical-activity-fr om-the-four-home-countries-chief-medical-officers (accessed 15 Dec2015).

Stodden, D. (2008). A Developmental Perspective on the Role of Motor Skill Competence in Physical Activity: An Emergent Relationship. Quest ; 60.doi:10.1080/00336297.2008.10483582.

Stratton, G., Jones, M., Fox, K. R., Tolfrey, K., Harris, J., Maffulli, N., ... Frostick, S. P. (2004). BASES Position Statement on Guidelines for Resistance Exercise in Young People. Jounal of Sports Science, 22. Recuperado a partir de http://research-information.bristol.ac.uk/en/publications/bases-position-statement-on-guidelines-fo r-resistance-exercise-in-young-people(df945d4e-702c-4d87-9488-9c3949036f68).html.

Treuth, M. S., Hunter, G. R., Figueroa-Colon, R., y Goran, M. I. (1998). Effects of strength training on intraabdominal adipose tissue in obese prepubertal girls. Medicine and Science in Sports and Exercise, 30(12), 1738-1743.

Vicente-Rodríguez, G. (2006) How does exercise affect bone development during growth? Sports Med Auckl NZ; 36:561-9.

Viciana, J., Mayorga-Vega, D., Cocca, A. (2013) Effects of a maintenance Resistance Training program on muscular strength in schoolchildren. Kinesiology; 45:82-91.

Watts, K., Jones, T. W., Davis, E. A., \& Green, D. (2005). Exercise training in obese children and adolescents - Current concepts. Sports Medicine, 35(5), 375-392. http://doi.org/10.2165/00007256-200535050-00002.

World Health Organization. (2010) Global recommendations on physical activity for health, Geneva; WHO. Recuperado a partir de: http://www.ncbi.nlm.gov/books/NBK305057.

Wolfe, R, R. (2006) The underappreciated role of muscle in health and disease. Am J Clin Nutr; 84:475-82.

Yu, C. C. W., Sung, R. Y. T., So, R. C. H., Lui, K.-C., Lau, W., Lam, P. K. W., y Lau, E. M. C. (2005). Effects of strength training on body composition and bone mineral content in children who are obese. Journal of Strength and Conditioning Research / National Strength \& Conditioning Association, 19(3), 667-672. http://doi.org/10 $.1519 / 14994.1$. 